\title{
Lapurdum
}

Euskal ikerketen aldizkaria | Revue d'études basques |

Revista de estudios vascos | Basque studies review

$6 \mid 2001$

Numéro VI

\section{Et... le hérisson se réveilla: Passé et présent de la littérature basque}

Mari Jose Olaziregi

\section{OpenEdition}

Journals

Édition électronique

URL : http://journals.openedition.org/lapurdum/1208

DOI : 10.4000/lapurdum.1208

ISSN : 1965-0655

Éditeur

IKER

Édition imprimée

Date de publication : 1 octobre 2001

Pagination : 205-217

ISBN : 2-84127-156-0

ISSN : $1273-3830$

Référence électronique

Mari Jose Olaziregi, «Et... le hérisson se réveilla : Passé et présent de la littérature basque », Lapurdum [En ligne], 6 | 2001, mis en ligne le 01 juin 2009, consulté le 31 janvier 2020. URL : http:// journals.openedition.org/lapurdum/1208; DOI : 10.4000/lapurdum.1208 


\section{Mari Jose Olaziregi Alustiza}

(Université du Pays Basque. Vitoria)

\section{Et... le hérisson se réveilla : Passé et présent de la littérature basque}

\section{Abstract}

Ce court article essaie de réaliser une révision de l'histoire de la littérature basque et de présenter un diagnostic de notre actualité littéraire. Pour ce faire, nous sommes partis de quelques vers de l'écrivain Bermardo Atxaga dans lesquels il compare notre littérature à un hérisson qui a vécu une trop longue léthargie, mais qui, heureusement, a réussi à s'éveiller au XX $X^{\mathrm{e}}$ siècle. Les caractéristiques de la langue basque, les débuts tardifs de notre littérature ou les allusions aux divers cadres politiques où elle se développe, constituent des aspects qui peuvent intéresser les lecteurs qui, ne parlant pas le basque, souhaitent découvrir notre littérature. L'article résume en suite les tendances qui ont marqué le développement du genre poétique et de la narration basques au XXe siècle. La Génération de poètes post-symbolistes de la décennie des années 30 , le relais de la poésie sociale à l'initiative de G. Aresti, ou l'irruption des idées postavant-gardistes à la fin des années 70, ne représentent que quelques arrêts dans notre itinéraire. Quant à la narration, nous allons parler de la prépondérance de la poétique de mours dans la première moitié du siècle, de l'irruption, dans les années 60, du roman moderne selon des typologies aussi distinctes que le roman existentialiste ou le roman expérimental proche du Nouveau Roman, du renouveau des récits courts, les contes, dans les années 80 , ou du panorama narratif actuel extrêmement varié. Nous complétons notre donne littéraire avec l'approche de l'auvre de notre auteur le plus international, Bernardo Atxaga. 
This short article attempts to give an overview of the history of Basque literature and make a diagnostic of our current literary scene. To that end, we have borrowed a few lines by the author Bernardo Atxaga in which he makes a comparison between our literature and a hedgehog that has been lethargic for too long, but has fortunately managed to wake up in the twentieth century.

The peculiarities of the Basque language, the late awakening of our literature and the references to the various political scenarios in which is has developed may be of interest to non-Basque readers who want to become familiar with our literature. After a few introductory notes, the article goes over the main currents that have shaped up the Basque poetic and narrative genres throughout the twentieth century. The generation of post-symbolist poets in the thirties, the social poetry of which G. Aresti is the main exponent, or the post-avanguard approach at the end of the seventies are some of the steps that we have taken in our journey.

As far as the narrative production is concerned, the description of local customs (known as costumbrismo) dominated the first half of the century, with modern novel bursting in in the sixties with varieties ranging from existencial novels to experimental works close to the Nouveau Roman, followed by the renovation of the short narrative genre, ie the writing of stories, in the eighties, all of which has resulted in the current multicoloured narrative scene.

We complete this short description with a few ideas about the work of our most international author, Bernardo Atxaga. 


\section{Le sommeil fut long, la bibliothèque brève; Mais au XXe siècle, le hérisson se réveilla.}

\section{Bernardo Atxaga ("J'écris dans une langue bizarre")}

Le réveil du hérisson, ce mystérieux animal qui se roule en boule et hérisse ses piquants à l'approche du danger, sert parfaitement à symboliser le développement de la littérature en langue basque. Comme Bernardo Atxaga le suggère dans son poème, il s'agit d'un hérisson qui, après une trop longue léthargie, a heureusement réussi à s'éveiller au XXe siècle. La période la plus intéressante et remarquable de notre histoire littéraire se situe donc dans les cent dernières années, période qui fera l'objet de la plupart des lignes qui suivent. D'où les rares références à notre passé littéraire le plus reculé, car, depuis la parution en 1545 du premier livre en basque, le recueil de poèmes Linguae Vasconum Primitiae de B. Etxepare, seuls 101 livres ont été publiés avant 1879 , dont quatre seulement peuvent être considérés comme strictement littéraires. De ce fait, nous parlons d'une littérature tardive, d'une littérature qui n'a pas connu de conditions socio-historiques fort favorables à son développement et qui a été liée, cela va de soi, aux avatars de la langue qui la soutient : la langue basque.

Et puisque nous parlons de langue basque, il conviendrait de faire quelques précisions à son sujet, dans le but de mieux connaître, voire d'aimer notre passé et notre présent littéraires. Tout d'abord, rappelons que nous écrivons et percevons dans une langue très ancienne, pré-indoeuropéenne d'après les experts; même si la date exacte de son origine reste inconnue, la plupart des anthropologues, des historiens et des linguistes sont d'accord pour dire qu'au Néolithique on parlait déjà cette langue. Ensuite, précisons qu'il s'agit d'une communauté linguistique très réduite, constituée à l'heure actuelle par quelque 700000 individus habitant des deux côtés des Pyrénées. La frontière politique qui divise aujourd'hui le Pays Basque, ou Euskal Herria, entraîne à son tour une situation légale différente. Si, depuis l'adoption de la Constitution Espagnole de 1978, la langue basque a un statut de co-officialité, au même titre que l'espagnol, dans les deux communautés autonomes de la zone espagnole, il n'en est pas de même au Pays Basque français, où la langue basque ne jouit pas de ce caractère de langue officielle. Les conséquen- 
ces de cette inégalité sont facilement prévisibles : grâce à l'instauration de modèles d'enseignement bilingues ou aux aides à l'édition en langue basque, le panorama littéraire basque s'avère beaucoup plus fort et dynamique au Pays Basque espagnol que dans la zone continentale.

Mais cela n'a pas toujours été le cas : les premières publications de notre histoire littéraire ont quand même vu le jour dans le Pays Basque français. D'autres textes ont suivi celui d'Etxepare et constituent des tournants importants dans le développement de la littérature en langue basque : la traduction, en 1571, du Nouveau Testament et de quelques écrits calvinistes réalisés par J. de Leizarraga ainsi que la parution en 1643 de l'œuvre Gero de Pedro de Axular, considérée comme le fleuron de la prose ascétique en notre langue. La publication de textes d'édification et de traductions se poursuit et, au XVIII siècle, c'est au Pays Basque espagnol qu'il faut situer la renaissance des cuvres et des auteurs. En 1765 sont fondés la Société Royale Basque des Amis du Pays et le Royal Séminaire de Vergara. Soutenus par les idées de l'Illustration, des auteurs tels que Francisco Javier Maria Munibe, Comte de Peñaflorida, ont éveillé et ravivé l'ambiance culturelle de l'époque. Dans la période 1794-1808 nous voulons souligner le niveau atteint par les activités liées à la langue. À ce moment-là, l'éminent linguiste G. de Humboldt nous rend visite pour devenir ensuite le diffuseur du basque dans les cercles européens. Beaucoup d'autres se succèdent; à l'ombre du Romantisme, le Pays Basque et notre ancienne langue attirent aussi l'attention de créateurs et de curieux, tels que le poète anglais $W$. Wordsworth ou l'écrivain français P. Mérimée, qui choisit un personnage basque, Carmen, comme héroïne de son célèbre roman.

Quoi qu'il en soit, c'est dans la dernière décennie du siècle précédent que nous découvrons les premiers indices d'un esprit nouveau, un esprit qui bouleverse de fond en comble l'avenir de notre littérature. Aussi assistonsnous à la fin de la prépondérance des ouvrages d'édification et de formation religieuse, et l'éventail des genres littéraires s'élargit : la trajectoire de certains poètes tels que Bilintx ou Etxahun s'enrichit avec les apports de nouveaux auteurs tels que Arrese Beitia; les récits, notamment des romans, font irruption dans le panorama littéraire basque. La perte des fors à la suite de la deuxième guerre carliste (1873-1876) a marqué le début de ce que la critique a appelé la "Renaissance" de la littérature basque. C'est à cette époque-là qu'avec Sabino Arana sont jetées les bases du nationalisme basque; celui-ci 
à son tour exerce son emprise sur toute la littérature basque du premier tiers du $X^{\text {e }}$ siècle. La suprématie de l'idéologie nationaliste a deux effets sur la production littéraire des premières décennies du $\mathrm{XX}^{\mathrm{e}}$ siècle : d'une part elle est troublée par des objectifs extra-littéraires et d'autre part elle passe à côté du mouvement moderniste européen qui a tenté de bouleverser et le langage et les formes déjà éclatées de l'époque moderne. Nous parlons des écrivains qui ont souscrit au manifeste lancé en 1930 par le poète E. Pound : "Make it new ! " et dont les tons novateurs ne sont arrivés chez nous que le siècle étant déjà bien entamé, vers les années 60. Le roman basque qui fait ses premiers pas à la fin du XIX $\mathrm{X}^{\mathrm{e}}$ siècle sous la plume de D. Aguirre essaiera de peindre un monde idéalisé et essentialiste, éloigné des cités industrielles qui se sont formées dans le Pays Basque. Il s'agit, en fait, d'un roman à thèse conçu autour de trois axes principaux : la foi, le patriotisme et son caractère basque. Voici le modèle qui subsistera jusqu' aux années 50.

Parmi les autres genres, c'est sans doute la poésie qui l'emporte dans la première moitié du $\mathrm{XX}^{\mathrm{e}}$ siècle. Forte d'une tradition littéraire bien plus formelle que celle de la narration, la poésie post-symboliste qui trouve sa meilleure expression dans l'œuvre de J. M. Lizardi, Lauaxeta et Orixe, a essayé d'explorer les possibilités expressives de notre langue. La Guerre Civile espagnole (1936-1939) a fait des ravages dans la production littéraire basque. Au grand nombre de pertes et d'exilés il faut ajouter la forte répression exercée par les vainqueurs. Nous parlons d'une époque où les prénoms basques ont été interdits, de même que les inscriptions en basque sur les pierres tombales des cimetières; une époque où les citoyens, l'administration, la culture sont autant de milieux sur lesquels la censure du régime de Franco s'est exercée. On a affirmé que la génération de l'après-guerre a été l'une des plus importantes pour la littérature basque, car elle lui a offert ce dont elle avait le plus grand besoin : une certaine continuité. La poésie a été le genre le plus cultivé, parmi d'autres raisons, parce qu'il était plus facile de publier des poèmes isolés que des œuvres complètes et que dans les années 1940-1959 l'activité éditoriale régulière était presque impossible. Signalons, parmi les poètes de cette époque, Ion Mirande : il a transgressé l'esprit religieux dissimulé dans la poésie basque jusqu'aux années 50. Hétérodoxe et nihiliste, héritier de Poe et de Baudelaire et lecteur de Nietzsche, Mirande nous a aussi laissé un roman, La filleule (1970), une sorte de version basque de Lolita de Nabokov. Aussi bien J. Mirande que G. Aresti (1933-1975) appartenaient à ce qu'on est convenu d'appeler la "Génération de 56", génération qui a cherché à mettre à jour la littérature basque par le biais de l'in- 
corporation des propositions modernes des littératures européennes; elle a surtout tenu à débarrasser la littérature basque de son servilisme politique, religieux ou folklorique, pour qu'enfin, la fonction esthétique du fait littéraire l'emporte sur toutes les autres. Les événements qui se sont succédé au Pays Basque quelques années plus tard, dans les années 60, (développement industriel et économique, consolidation des écoles basques ou ikastolas, unification de la langue basque, activité politique importante contre le régime de Franco qui censurait toute activité culturelle en basque, campagnes d'alphabétisation en langue basque...), ont préparé un terrain propice à la germination des nouveaux principes littéraires. On a dit qu'on a opposé à l'orthodoxie culturelle en vigueur à l'époque une hétérodoxie culturelle et politique animée par des auteurs tels que le poète G. Aresti, le remarquable philologue K. Mitxelena (1915-1987) et le sculpteur J. Oteiza (1908). Après la publication de Maldan Behera [La descente] (1960) qui présente l'empreinte évidente de la sensibilitê d'Elliot, Gabriel Aresti s'est penché vers la poésie sociale à la suite de la parution de Harri eta Herri [Pierre et Peuple] (1964).

Quant aux récits, le roman existentialiste Leturiaren egunkari ezkutua [Le journal secret de Leturia] (1957) de Txillardegi marque le début de la modernité pour le roman écrit en basque. Quelques années plus tard, en 1969, lors de la publication de Egunero hasten delako [Parce que ça commence tous les jours], l'écrivain Ramón Saizarbitoria bouleverse de fond en comble la donne romanesque : un roman expérimental proche du Nouveau Roman français se substitue à la poétique existentialiste. Commence une nouvelle période où la forme romanesque et l'expérimentation l'emporteront dans les univers littéraires des auteurs de l'époque. Cet étalage formel bat son plein en 1976 avec la publication de Ene Jesús [Oh! mon Dieu], du même auteur. Dans les années 70, le plus international de nos auteurs fait irruption dans le panorama de la littérature basque : Bernardo Atxaga. Même si à ses débuts il a publié des ouvrages au goût post-avant-gardiste et de facture expérimentale, il a vite évolué vers des conceptions plus fantastiques ou réalistes. Étant donné que nous nous occuperons plus tard de son évolution littéraire, pour l'instant nous allons nous borner à compléter ce commentaire en disant que vers la fin des années 70 , tout comme dans les littératures contiguës, le roman basque a récupéré le goût du conte. La prémisse post-moderne "tout est déjà raconté mais il faut le rappeler" est à l'origine de beaucoup de textes des dernières décennies. 
Bien qu'il n'ait pas entraîné de bouleversement dans les paradigmes littéraires basques de l'époque, le début de l'ère démocratique espagnole en 1975 a permis de mettre en place des conditions objectives menant au plein développement de la littérature basque en tant qu'activité autonome. Les données de ce nouveau panorama sont éloquentes : actuellement on publie quelque 1500 nouveaux titres par an, il y a quelque 100 maisons d'édition sur le territoire du Pays Basque, environ 300 écrivains, dont $10 \%$ seulement sont des femmes, et la narration est le genre le plus cultivé, en particulier le roman, genre plébiscité par les lecteurs de la dernière décennie. D'un autre côté, à partir de 1981, on peut suivre des études universitaires de langue et littérature basque, ce qui permet le renforcement de la critique académique ainsi que la parution de nouvelles générations de chercheurs. On assiste à la consolidation d'événements importants, tels que la Foire annuelle du Livre de Durango. Les traductions d'œuvres universelles en basque connaissent un développement quantitatif et qualitatif inouï, au point que j'oserais affirmer qu'à l'heure actuelle on est en mesure de lire en basque sans problème des auteurs universels tels que Faulkner, Joyce, Kundera, Hasek, Skvoreck ?... Ce ne sont que des indicateurs qui dessinent un panorama éditorial assez consolidé, où la publication et la lecture de textes littéraires en basque a atteint des niveaux inconnus jusqu'à présent. Sans aucun doute l'aspect le plus faible de notre système littéraire est toujours le nombre réduit d'ouvrages écrits en basque qui sont traduits à d'autres langues. Sur les 60 titres qui ont été traduits en différentes langues, l'œuvre de B. Atxaga vient en tête non seulement à cause du nombre de langues dans lesquelles elle a été traduite (Obabakoak peut se lire en 25 langues différentes) mais aussi grâce à l'écho et au succès international connus par l'auteur. Malgré la remarquable infrastructure éditoriale, médiatique et académique dont nous disposons aujourd'hui, la littérature basque ne se montre toujours pas telle qu'elle est : une littérature en quête de nouveaux lecteurs.

Reprenons cette courte révision de notre littérature contemporaine. Dans les années 70 la poésie sociale d'Aresti est remplacée par une poésie existentialiste, telle que celle de X. Lete ou celle d'auteurs comme A. Urretabizkaia ou M. Lasa [Memory Dump] (1993). D'autres auteurs sont partis de positions post-symbolistes pour évoluer vers un style plus concentré et synthétique (J. M. Lekuona), ou bien vers l'instrospection personnelle. (B. Gandiaga). K. Izagirre entame aussi sa trajectoire poétique dans les années 70: Itsaso Ahantzia [La mer oubliée] (1976) est un texte proche de l'esthétique surréaliste, pour évoluer vers une poésie engagée en 1989, avec 
la parution de Balizko erroten erresuma [Le royaume des moulins fictifs]. Pour sa part, J. Sarriaonandia, après le recueil de poèmes riches de références culturelles Izuen gordelekuetan barrena [À travers les cachettes de la peur] (1981), un voyage littéraire où il puisait surtout dans Kavafis, Holan et Pessoa, il s'est approché aussi d'une poétique plus engagée dans Marinel zaharrak [Les vieux marins] (1987) et Huny illa nyha majah yahoo (1995).

Mais le livre qui a vraiment bouleversé la donne poétique de l'époque est Etiopia (1978), de Bernardo Atxaga : ce livre a établi le canon de la poésie moderne parmi nous. La parution de l'ouvrage a eu lieu à une époque où la poésie basque connaît sa période d'avant-garde, grâce à la prolifération de revues littéraires qui ont joué le rôle de plaque tournante pour beaucoup d'auteurs. Pour compléter cette présentation, il nous reste à signaler que dans les années 80 nous assistons à une pluralité de tendances poétiques, dont la consolidation de la poésie dite de l'expérience. Des poètes de Ia taille de F. Juaristi (Denbora, nostalgia) [Temps, nostalgie] (1985), Galderen geografia [Géographie des questions] (1997), A. Iturbide, J.K. Igerabide ou M. J. Kerexeta, vont combiner une approche au symbolisme et à l'esthéticisme et le recours à l'expérience personnelle, en tant que base de leur poésie personnelle. D'autres auteurs, tels que T. Irastorza, ont publié des poèmes plus intimistes. Malgré la diminution du nombre d'ouvrages poétiques publiés dans les années 90, d'autres auteurs se sont incorporés à notre panorama : R. Diaz de Heredia (Hari hauskorrak) [Les fils fragiles] (1993), Kartografia (1998) ou G. Markuleta: D'un autre côté, les écrivains groupés autour de la revue Susa, auteurs d'une poésie rupturiste, underground pour ainsi dire, ont assisté à l'élargissement de leur groupe initial de poètes (Izagirre, Aranbarri, Nabarro, Montoia, Otamendi, Borda...) grâce à l'incorporation d'autres noms dans les années 90 (J. Olasagarre, H. Cano, G. Berasaluze...).

Quant à la narration, genre nettement prépondérant dans les dernières décennies, signalons que la prolifération de revues littéraires dans les années 80 a contribué au développement du conte moderne du fait de leur brièveté. Les ouvrages de J. Sarrionandia (Narrazioak) (1983) ou de Atxaga, notamment son excellent livre Obabakoak (1988), nous emmènent à des mondes fantastiques et imaginaires, auparavant inconnus dans la prose écrite de notre langue. Cette trajectoire du conte moderne s'est vue confirmée par les narrations portant l'empreinte de Rulfo de I. Mujika Iraola dans Azukrea belazeetan [Du sucre dans les prés] (1987), ou par le réalisme sale de X. 
Montoia. Quoi qu'il en soit, comme il arrive dans la littérature espagnole de la fin du siècle, l'activité littéraire basque a aussi tourné autour du roman dans les derniers temps. À présent la narration est le genre qui connât le plus grand succès, le plus grand prestige et offre, bien sûr, la plus grande rentabilité éditoriale. Si nous devions présenter brièvement les tendances et les auteurs les plus remarquables d'aujourd'hui, nous devrions commencer par les auteurs qui adhérent au roman lyrique ou poétique; celui-là a commencé à proliférer à la fin des années 70 et parmi ses représentants se comptent le texte intimiste et proche du "féminisme de la différence" des années 70 : Zergatik Panpox [Pourquoi panpox?], (Orain, 1995) de A. Urretabizkaia, ou: les romans de Juan Luis Zabala: Zigarrokin ziztrin baten azken keak [La fumée d'un mégot insignifiant] (1985) et Kaka esplikatzen [Bagout] (1989). Ces ouvrages rappellent le goût pour le détail symbolique de Hanhka ou le désespoir omniprésent dans l'univers de T. Bernhard. Un autre genre romanesque qui foisonne chez nous ces derniers temps est bien le roman policier sous ses divers aspects. Par ailleurs, si dans la plupart des romans publiés. dans les années 70 prédominaient la préoccupation linguistique et les modèles classiques anglais du whodunit, à partir des années 80 d'autres éléments. du thriller contemporain et du roman noir américain s'y sont progressivement: incorporés. Pour ne citer que quelques titres intéressants, voilà le roman d'espionnage : Izua hemen [La peur ici] (1991) de J.M. Iturralde; l'excellent: roman Ur uherrak [De l'eau trouble] (Hiru, 1995) de A. Epaiza; El hombre solo [L'homme seul] (1994), de B. Atxaga, thriller psychologique qui a remporté plusieurs prix; l'inquiétante histoire de mystère Katebegi galdua [Le maillon perdu] (1996) de Jon Alonso ou les romans à rebondissements Beluna Jazz (1996) et Pasaia Blues (1999) de Harkaitz Cano.

Outre les caractêristiques signalées, le réalisme, l'observation de la réalité extérieure règnent dans les ouvrages de narration les plus récents. Il s'agit d'un réalisme agrandi par les nouvelles perspectives et points de vue, par leur rayonnement fabulateur ou par le traitement formel. Ce regard vers l'extérieur est à l'origine de la prolifération des romans autour d'événements historico-politiques importants pour notre histoire contemporaine. En plus. des romans réalistes signalés de $\mathrm{B}$. Atxaga, nous avons les dernières publications de R. Saizarbitoria : Los pasos incontables [Les pas incalculables] (1998) et Amor y guerra [Amour et guerre] (1999), où la mémoire joue le rôle d'axe narratif autour duquel il écrit une sorte de roman de témoignage. Pour compléter ce puzzle il faut parler de la trajectoire importante de certains auteurs tels que J.M. Irigoien, qui a apporté des airs sudaméricains à nos 
romans (Babylone) (1998), ou encore du perpétuel renouveau poétique qui est à l'origine de toute la production de A. Lertxundi. Ses débuts néorréalistes (Goiko kale) (1973) ont évolué vers des univers littéraires qui ne s'inspirent plus d'événements réels mais de simples conjectures littéraires. Avec Lertxundi nous entamons un voyage littéraire, un voyage intertextuel, qui se nourrit de différentes traditions poétiques. Les romans Las últimas sombras (1996) [Les dernières ombres] et Un final para Nora (1999) [Une fin pour Nora] en sont des exemples.

\section{Bernardo Atxaga (1951) : charmeur de lecteurs.}

Il n'est peut-être pas très orthodoxe de parler de José Irazu Garmendia, connu sous son nom d'auteur de "Bernardo Atxaga", comme d'un charmeur de lecteurs. Mais les mots charme et séduction pourraient bien décrire à eux seuls le succès que connaît chez nous notre auteur le plus universel. Étant donné l'incontestable mercantilisation de la littérature, l'expression "l'auteur le plus lu" peut nous induire en erreur. Pourtant Bernardo Atxaga est bel et bien l'auteur plébiscité par le public, l'auteur qui vend le plus d'ouvrages écrits en basque, le plus apprécié par les autres écrivains; enfin l'auteur devant lequel s'incline la critique académique basque. D’ailleurs, tous ceux qui ont assisté à l'une de ses lectures poétiques ou à ses conférences connaissent son dévouement, son empressement envers ses lecteurs. Cette attitude artistique a contribué à ce que la littérature de B. Atxaga fasse partie de nos vies. C'est pourquoi il n'est pas rare aujourd'hui de lire des titres de presse évoquant l'un de ses ouvrages, ni de voir des B.D. humoristiques montrant l'un de ses personnages. La phrase de la petite chienne Shola dans Pò̀mes et Hybrides ("personne n'est apprécié chez soi") n'est plus vraie pour Atxaga. C'est aussi sans doute cet attrait et ce charme que suscite son œuvre qui sont à l'origine du livre intitulé Le sourire de Bernardo Atxaga publié par Dinapiera di Donato au Venezuela et de ce que le journal anglais The Guardian compte Atxaga parmi les 21 meilleurs écrivains du $\mathrm{XX}^{\mathrm{e}}$ siècle.

En reprenant ses propres termes, nous pourrions présenter son œuvre littéraire comme un inventaire qui comprendrait trois grands volets. D'un côté, les textes fantastiques situés à Obaba. D'un autre côté, les romans réalistes qui prennent les personnages pour trame narrative. Et enfin toute une série d'ouvrages où se manifeste son aspect d'avant-garde, le plus innova- 
teur : la littérature pour enfants et pour les jeunes, les alphabets ou les ouvrages poétiques tels que Poèmes et Hybrides. Même si les dimensions de cet article ne nous permettent pas de trop nous occuper de chacun d'entre eux, nous essaierons d'en préciser les principales caractéristiques.

Commençons par Obaba, cette géographie imaginaire qui confère son unité à tout un cycle fantastique comprenant des contes et des récits publiés dans les années 80 tels que Lorsqu'un serpent... (1984), Deux lettres (1984), le roman Deux Frères (1985), ou le célèbre livre de récits Obabakoak (1988). Si ce topo littéraire a pour origine une berceuse biscayenne, ses descriptions nous parlent d'un paysage affectif plus large, d'un paysage relié au passé, à un monde ancien. Il s'agit d'une géographie vécue qui fuit toute concrétisation topologique et dans laquelle règne la causalité magique. (cf. J.L. Borges). Dans ce monde primitif les personnages se servent des animaux pour exprimer divers malheurs ou diverses situations. C'est pourquoi il n'y a rien d'étonnant à ce que ses habitants croient dur comme fer qu'un enfant puisse devenir un sanglier blanc, ni à ce que les lézards verts soient des êtres dangereux qui pourraient faire des ravages dans nos têtes s'ils réussissaient à s'y introduire par nos oreilles. Par le biais de ces superstitions, si présentes dans notre tradition orale, Atxaga nous parle de sentiments aussi universels que la peur, ou de la lutte perpétuelle entre la nature et la civilisation. Ainsi, dans Obabakoak (1988), excellent livre traduit en 25 langues, qui a reçu de nombreux prix, dont le Prix National de Narration- 1989, Atxaga nous présente un voyage littéraire qui, à partir de motifs et d'éléments bel et bien basques (particuliers), rend hommage aux maîtres universels du conte littéraire. Cet hommage littéraire est rendu par le biais des citations de contes (par exemple, le célèbre Le serviteur du riche marchand), des résumés (tels que ceux des contes de Tchekhov, Waugh et Maupassant dans $\grave{A}$ propos des contes), des paraphrases aux transformations thématico-formelles (telles que Wei Lie Deshang), des plagiats (comme dans le conte Torture par espérance de Villiers de l'Isle Adam dans Une crevasse dans la neige gelée) des parodies, des imitations, etc. Au cas où ces références ne seraient pas suffisantes, des titres tels que Margarete et Heinrich, jumeaux (cf. G. Trakl) ou E. Werfell (cf. F. Werfel) nous parlent des racines expressionnistes de certains contes du livre. 
Dans le délicieux roman qui raconte les souvenirs de la vache Mo, Mémoires d'une vache (1991), Atxaga annonce les caractéristiques de ses romans postérieurs : le réalisme et l'utilisation de voix intérieures pour raconter le discours intérieur des personnages. Traduit en 13 langues, le roman L'homme seul (1993), tourne bien autour d'un personnage (Charles) et de l'impitoyable solitude qu'il éprouve. Hanté par les voix qu'il entend au fond de son âme ainsi que par les souvenirs du passé, Carlos, ancien activiste de l'E.T.A., ne défend plus les idéaux révolutionnaires d'antan. L'image surréaliste de la mer glacée qui ne quitte jamais le protagoniste de cet inquiétant thriller traduirait parfaitement l'angoisse qu'il éprouve en se sentant perpetuellement traqué. Tout comme dans les romans de Pavese, dans L'homme seul le lecteur saisit l'impitoyable sentiment de solitude qui entoure les personnages principaux. Quelques années plus tard, en 1995, Atxaga publiait Ciels, roman court où l'on raconte le voyage de retour d'une ancienne militante réinsérée de l'ETA, Irène. À nouveau nous avons affaire à un personnage déçu et solitaire qui essaie de s'éloigner d'un passé qui le rend prisonnier. Mais cette fois-ci c'est la littérature qui va permettre à Irène de s'éloigner de ce sentiment de solitude qui la saisit. L'excellente anthologie poétique que comporte le roman nous rappelle ce vers célèbre de $\mathrm{E}$. $\mathrm{M}$. Cioran : "Y a-t-il de critère artistique sans s'approcher des ciels?". D'où l'importance accordée dans ce roman à la symbologie des ciels : un ciel où se reflète parfois Irène et qu'elle désire "toucher".

Peu à peu nous arrivons au dernier grand volet de l'œuvre d'Atxaga, formé par ses textes d'avant-garde. Nous y découvrons un auteur plus novateur et hardi, qui aime faire des expérimentations avec des engins littéraires tels que les textes pour enfants, les alphabets ou abécédaires, ou qui n'hésite pas à proposer des lectures poétiques publiques à la manière de Dylan Thomas.

Comme nous l'avons dit plus haut, la publication du recueil de poèmes Etiopia (1978) a marqué une date dans le développement de la poésie basque moderne. Il s'agit d'un collage de livres de récits et de poèmes, organisés dans une structure circulaire. Deux récits ouvrent et ferment le livre et, au milieu, imitant le célèbre texte de Dante, nous trouvons neuf cercles de sable délimitant ce voyage littéraire vers Etiopia (Utopie). Et c'est sur le sable que le temps oublie des montres cassées; la mémoire, des lettres jaunâtres; la culture, un dictionnaire lilliputien et le courage, un bouclier rouillé (réf. "À propos du 
sable"). En fait ce qui se dégage d'Etiopia, c'est bien la lassitude poétique qui a précédé la fin de la modernité. Atxaga tente de bouleverser le langage poétique corrompu et nous annonce l'arrivée de wagons pleins de silence qui luttent contre les substantifs et les adverbes (réf. "Chronique partielle des années 70"). Le protagoniste est un apatride empreint de l'esprit Dada et qui cherche à imiter Rimbaud. Ainsi flâne-t-il dans cette ville dramatique (et nettement expressionniste). Devenue un terrible labyrinthe, la ville est peuplée d'anti-héros et de perdants (tels qu'explorateurs, boxeurs) ou... de poètes suicidaires. Les camarades de voyage invoqués par le poète (Nerval, Rigaut, Rimbaud ou Cravan) ont aussi cherché à fuir la Ville, c'est-à-dire, les limites du langage.

Débarrassé des excès et de l'ornement, éloigné du dramatisme qui règne dans le livre précédent, dans Poèmes et Hybrides (1990) Atxaga essaie de récupérer le sens essentiel de la poésie. Pour ce faire, il déchire le langage topique dont la poésie se servait, en le brisant à l'aide du dadaïsme et à l'aide d'éléments primitifs et enfantins. Humour et tendresse président l'univers de l'auteur, mais c'est surtout le besoin de s'éloigner de toute conception élitiste du travail poétique qui l'emporte. Les personnages de Poèmes et Hybrides tels que Ainhoa avec sa robe fraise et vanille (réf. "Famille III"), la "mystérieuse" Shola (réf. "Famille IV"), ou l'immigrant Ezekiel Masisi Dembele (réf. "Chansons IX") témoignent du regard humaniste et engagé d'Atxaga. Sa quête de nouvelles possibilités expressives comprend des chansons, des lectures poétiques sous forme d'inventaire (réf. Henry Bengoa Inventarium), voire des peintures, dont le charme a fait de Nouvelle Etiopia (1998) un livre vraiment séducteur. De n'importe quel point de vue qu'on l'observe, le réveil du hérisson s'avère extrêmement intéressant. 